\title{
Ultrafast Charge Transfer Dynamics at the Origin of Photoconductivity in Doped Organic Solids
}

\author{
Published as part of The Journal of Physical Chemistry virtual special issue "125 Years of The Journal of \\ Physical Chemistry".
}

Demetra Tsokkou, Chiao-Yu Cheng, Nina Krainova, Sukrit Mukhopadhyay, Noel C. Giebink, and Natalie Banerji*

Cite This: J. Phys. Chem. C 2021, 125, 7086-7096

Read Online

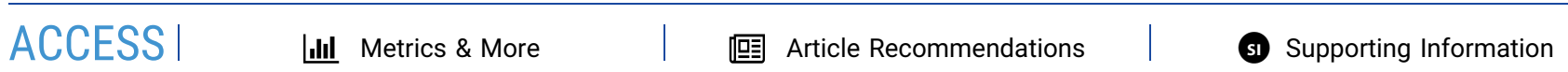

ABSTRACT: In spite of their growing importance for optoelectronic devices, the fundamental properties and photophysics of molecularly doped organic solids remain poorly understood. Such doping typically leads to a small fraction of free conductive charges, with most electronic carriers remaining Coulombically bound to the ionized dopant. Recently, we have reported photocurrent for devices containing vacuum-deposited TAPC (1,1-bis(4-bis(4methylphenyl)aminophenyl)cyclohexane) doped with $\mathrm{MoO}_{3}$, showing that photoexcitation of charged TAPC molecules increases the concentration of free holes that contribute to conduction. Here, we elucidate the excited-state dynamics of such

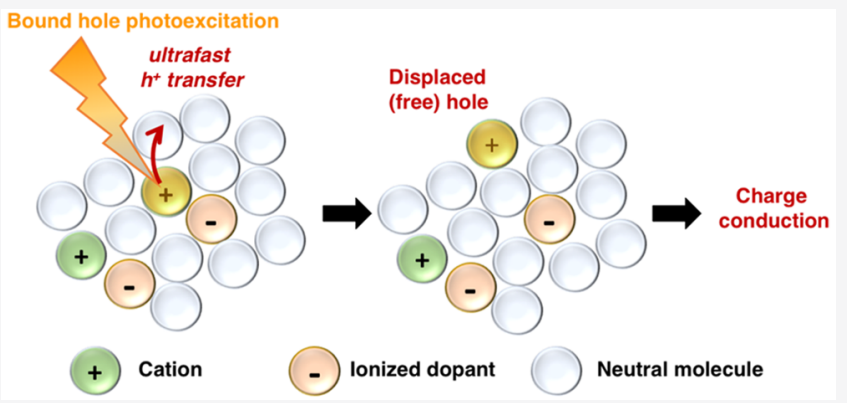
doped TAPC films to unravel the key mechanisms responsible for this effect. We demonstrate that excitation of different electronic transitions in charged and neutral TAPC molecules allows bound holes to overcome the Coulombic attraction to their $\mathrm{MoO}_{3}$ counterions, resulting in an enhanced yield of long-lived free carriers. This is caused by ultrafast back-and-forth shuffling of charges and excitation energy between adjacent cations and neutral molecules, competing with relatively slow nonradiative decay from higher excited states of $\mathrm{TAPC}^{\bullet+}$. The light-induced generation of conductive carriers requires the coexistence of cationic and neutral TAPC, a favorable energy level alignment, and intermolecular interactions in the solid state.

\section{INTRODUCTION}

Molecular doping increases the conductivity of organic solids by several orders of magnitude. ${ }^{1-4}$ Such materials are advantageous transporting layers in various electronic devices and have been commercially applied in displays. Despite being known for decades, ${ }^{5,6}$ the mechanisms underlying free charge generation in doped organic semiconductors have only recently begun to be understood. ${ }^{7-12}$ In general, doping is achieved by blending a host material with a strong molecular dopant to form radical ions via charge transfer between the two materials. However, many of the resulting electronic charge carriers (i.e., electrons and holes) remain bound in the Coulomb potential of their ionized dopant counterions, limiting the conductivity of the film. ${ }^{1,2}$ One way of exploring the dissociation of these charge pairs is to optically excite the charge carriers, giving them additional energy to escape, similar to the pump-push-probe studies used to study charge transfer state dissociation in organic photovoltaic donoracceptor blends. ${ }^{13-15}$ In this context, we recently demonstrated an increase in photocurrent external quantum efficiency when exciting the optical transitions of doped small molecule thin film devices, suggesting that photoexcitation can assist free carrier generation. ${ }^{16}$ However, the detailed origin of this effect is not yet resolved.

So far, photoexcited radical ions have been of interest because of their increased reactivity in the excited state, for example, in artificial photosynthesis. ${ }^{11,12}$ Ultrafast transient studies are necessary to investigate their extremely short-lived excited-state dynamics ${ }^{12,17}$ and have revealed that charge transfer from photoexcited radical ions can occur either intramolecularly in donor-acceptor dyads or intermolecularly between different molecules in solution. ${ }^{11,12,18,19}$ However, to the best of our knowledge, such studies are scarce for doped organic materials in the solid state, ${ }^{7,20}$ where additional intermolecular interactions become significant. Here, we employ femtosecond transient absorption (TA) spectroscopy on TAPC films doped with $\mathrm{MoO}_{3}$ to probe their excited-state

Received: March 5, 2021

Revised: March 10, 2021

Published: March 29, 2021 
(a)

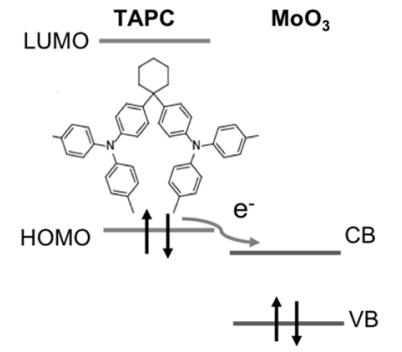

(b)

TAPC $^{-+}$cation

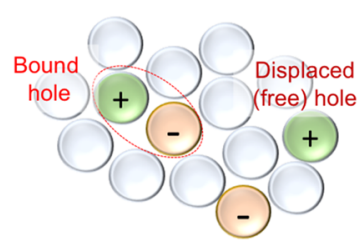

Neutral TAPC

$\mathrm{TAPC}^{*+}$ cation

$\mathrm{MoO}_{3}$ clusters

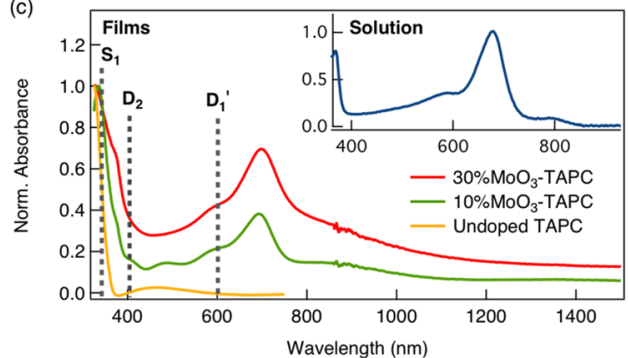

(d) Neutral TAPC

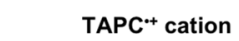
(removal of $\beta$-electron)

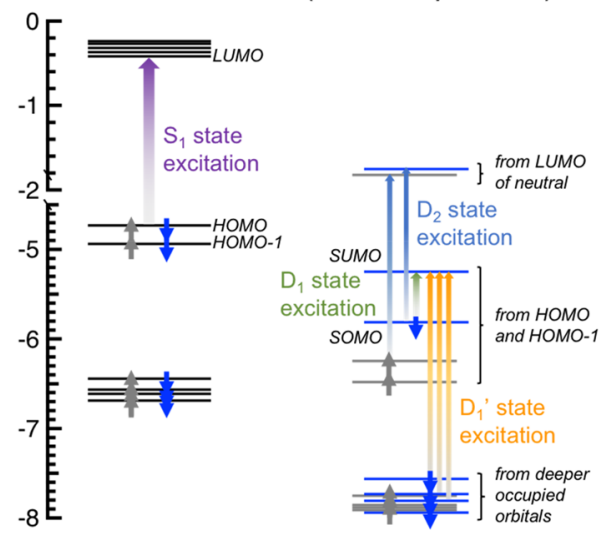

Figure 1. Absorbance and energetics. (a) Frontier energy levels and electron transfer from $\mathrm{TAPC}$ to $\mathrm{MoO}_{3}$, resulting in radical cations on TAPC. The chemical structure of TAPC is included. (b) Schematic illustration of the molecular packing in doped TAPC films including bound holes that are strongly Coulombically bound to the ionized dopant and displaced (free) holes on TAPC ${ }^{\bullet+}$ cations that have a larger spatial separation from the ionized dopant and therefore weaker interactions. (c) Normalized steady-state absorbance spectra of undoped TAPC, doped $10 \%$ MoO $3-\mathrm{TAPC}$, and $30 \% \mathrm{MoO}_{3}-\mathrm{TAPC}$ films and $\mathrm{TAPC}^{\bullet+}$ solution in $\mathrm{H}_{2} \mathrm{SO}_{4}$ (inset). The dotted vertical lines represent the excitation wavelengths used in transient absorption measurements that correspond to transitions to the $S_{1}$ state $(340 \mathrm{~nm}), \mathrm{D}_{2}$ state $(400 \mathrm{~nm})$, and $\mathrm{D}_{1}{ }^{\prime}$ state $(600 \mathrm{~nm})$. (d) Orbital energy levels and optical transitions of TAPC and TAPC ${ }^{\bullet+}$ predicted by DFT and TD-DFT calculations. For the radical cation, the electrons with $\alpha$-spin (gray) and $\beta$-spin (blue) have different energies, as indicated by the spin-orbital levels. We also show from which orbitals of neutral TAPC the ones of $\mathrm{TAPC}^{\bullet+}$ originate.

dynamics in comparison to $\mathrm{TAPC}^{\bullet+}$ in solution. We find that pumping different transitions of $\mathrm{TAPC}^{\bullet+}$ in the films systematically leads to ultrafast hole transfer (HT) to adjacent neutral TAPC molecules that coexist with the cations in the solid state. In this manner, photoexcitation of the cations helps to dissociate holes that are bound in the Coulomb potential of their $\mathrm{MoO}_{3}$ dopant counterions, explaining the photocurrent that we have previously observed. ${ }^{16}$ Moreover, regardless of whether cations or neutral molecules are initially photoexcited, charge and/or excitation energy transfer between them ultimately results in the formation of free holes in TAPC that are characterized by a long-lived ( $>1 \mathrm{~ns}$ ) electroabsorption (EA) signature.

Our findings provide new fundamental insights about the photophysics of radical ions in the solid state and are generally relevant to understanding separation of bound charges in doped organic solids and organic solar cells.

\section{EXPERIMENTAL SECTION AND COMPUTATIONAL DETAILS}

Fabrication and Characterization. Film deposition was performed in a vacuum thermal evaporator by coevaporation of TAPC and $\mathrm{MoO}_{3}$ with a base pressure of $5 \times 10^{-8}$ Torr. 1,1Bis $[4-[N, N$-di(p-tolyl)amino $]$ phenyl $]$ cyclohexane (TAPC, 97\% purity) was purchased from Sigma-Aldrich and purified once via gradient sublimation. Molybdenum(IV) oxide (99.9995\% purity) was purchased from Alfa Aesar and used as received. For these experiments the samples were deposited on a glass substrate. The absorption spectra of the films were measured with a Lambda 950 UV-vis-NIR spectrophotometer (PerkinElmer) via the use of an integrating sphere. Doped TAPC solutions were prepared by adding the corresponding undoped powder to sulfuric acid. The solution concentration was about $\sim 5 \times 10^{-4} \mathrm{M}$, while the maximum cation-related absorbance band in the visible region was around 0.2.

Transient Absorption (TA) Spectroscopy. To study the excited-state dynamics, spectrally resolved femtosecond TA measurements were performed by using a Ti:sapphire amplifier system (Astrella, Coherent). The output pulses had a time duration of $\sim 35 \mathrm{fs}$, an $800 \mathrm{~nm}$ center wavelength, a repetition rate of $1 \mathrm{kHz}$, and an energy of $\sim 6 \mathrm{~mJ} /$ pulse. Part of the amplifier output was used to pump the optical parametric amplifier (OPA) (Opera, Coherent) that converted the photon energy of the incident beam to the wavelength used to photoexcite the samples. Pump wavelengths at 340, 400, 600, and $730 \mathrm{~nm}$ were used. Probe wavelengths covering the visible and near-infrared (NIR) region ranging from 480 to $1300 \mathrm{~nm}$ were spectrally resolved. This was achieved via continuum white light pulses generated by strongly focusing a small part of the fundamental beam onto a $5 \mathrm{~mm}$ sapphire plate. Part of the probe pulses was then temporally and spatially overlapped on the sample with the pump pulses, while the other part was used as a reference. The transmitted probe beam through the sample and the reference beam were spectrally dispersed in 
two home-built prism spectrometers (Entwicklungsbüro Stresing, Berlin) and detected separately with either backthinned silicon CCDs (Hamamatsu S07030-0906) or InGaAs arrays (Hamamatsu) for respectively visible and NIR detection. The transmission change of the probe pulses following photoexcitation was recorded for different pumpprobe time delays up to nanoseconds, while the pump pulses were chopped at $500 \mathrm{~Hz}$ for the signal to be measured shot by shot. The TA changes induced by the pump were monitored with $\approx 70$ fs time resolution. The beam sizes of the excitation and probe pulses were $\approx 1 \mathrm{~mm}$ and $250 \mu \mathrm{m}$, respectively, to ensure uniform distribution of detected photoexcited species. To avoid anisotropy effects, the relative polarization of the probe and pump pulses was set at the magic angle. The TA spectra for the entire time window were scanned multiple times for both films and solution without any significant signs of degradation. For solid-state measurements, the films were sealed in a chamber filled with nitrogen, while the solution was placed in a quartz cuvette with an optical path length of $2 \mathrm{~mm}$. All TA data were corrected for the chirp of the white light.

DFT Calculations. The structures of isolated TAPC molecule in the ground state and cationic state are optimized by using density functional theory (DFT) at the B3LYP/6$31 \mathrm{~g} * *$ level. ${ }^{21-26}$ The vibrational analysis on the ground-state geometries is performed, and the lack of imaginary frequencies is used to ascertain the minima in the potential energy surface (PES). The ground-state geometries and excited states of the isolated radical cation are computed by using the unrestricted method. The effect of dielectric medium $(\varepsilon=2.5)$ is incorporated by the conductor-like polarizable continuum model $(\mathrm{cpcm}) .{ }^{27}$ The excited-state energies are computed by using time-dependent density functional theory (TD-DFT). The nature of a few excited states was analyzed by using natural transition orbitals (NTIOs). ${ }^{28}$ These calculations were performed by using the G16 suite of programs. ${ }^{29}$

\section{RESULTS AND DISCUSSION}

The molecule of interest in this study is TAPC, composed of two triphenylamine moieties (Figure 1a), and well-known as a hole-transporting material used in organic light-emitting devices. ${ }^{30,31}$ Its advantageous properties include stability, high hole mobility $\left(10^{-1}-10^{-2} \mathrm{~cm}^{2} /(\mathrm{V} \mathrm{s})\right),{ }^{24,32}$ and a high ionization potential $(\sim 5.5 \mathrm{eV}) .^{30,31}$ Here, we generate TAPC $^{\bullet+}$ in solution by dissolving TAPC powder in concentrated sulfuric acid $\left(\mathrm{H}_{2} \mathrm{SO}_{4}\right)$. Alternatively, p-doped TAPC films are formed by thermally coevaporating TAPC with $\mathrm{MoO}_{3}$ (electron affinity of $\sim 6.7 \mathrm{eV}$ ). ${ }^{3-35}$ This energetic arrangement induces electron transfer (ET) from the HOMO of TAPC to the deep-lying conduction band of $\mathrm{MoO}_{3}$ (Figure 1a). ${ }^{33}$ Thus, radical cations are generated in TAPC that are counterbalanced by $\mathrm{MoO}_{3}$ anions (Figure $1 \mathrm{~b}$ ). Because of the strong Coulombic interaction within these ion pairs (hundreds of millielectronvolts), most of the resulting holes in TAPC remain bound to their $\mathrm{MoO}_{3}$ counterions, as evident from the large discrepancy between hole concentration inferred from optical absorption and electrical conductivity measurements. ${ }^{2,9,36}$

Figure 1c shows the normalized absorbance spectra of vacuum-deposited undoped TAPC and two doped TAPC films with different $\mathrm{MoO}_{3}$ concentrations of 10 vol $\% \mathrm{MoO}_{3}$-TAPC and 30 vol $\% \mathrm{MoO}_{3}$-TAPC. All films exhibit absorption below $380 \mathrm{~nm}$, related to the $S_{0} \rightarrow S_{1}$ transition of neutral TAPC molecules. ${ }^{37}$ The involved orbital energy levels are shown in Figure 1d, while the corresponding frontier orbitals are depicted in Figure S1. The transition is dominated by the excitation of an electron from the nearly degenerate higherlying occupied orbitals (HOMO and HOMO-1) to low-lying unoccupied orbitals (LUMOs) according to our density functional theory (DFT) calculations (Figure S2). The weak band between 400 and $550 \mathrm{~nm}$ in undoped TAPC film was previously attributed to TAPC aggregates and/or singlettriplet intermolecular charge transfer excitons formed between TAPC subunits, although we do not exclude interference effects to be the origin of the signature. ${ }^{37}$ In the doped films, the absorption in the visible range (peaking at $\sim 700 \mathrm{~nm}$ ) and between 350 and $400 \mathrm{~nm}$ is assigned to TAPC ${ }^{\bullet+}$. Similar bands have been reported for chemically oxidized TAPC with $\mathrm{FeCl}_{3}$, ${ }^{38}$ electrochemically oxidized TAPC, ${ }^{26}$ and photoexcited $\mathrm{C}_{60}$ /TAPC blends. ${ }^{38-40}$ TAPC $^{\bullet+}$ in solution has comparable absorption bands as in the doped films (inset of Figure 1c). The slight blue-shift of the solution maximum $(\sim 680 \mathrm{~nm})$ compared with that in thin films is related to environmental effects including reduced intermolecular interactions and solvation. The increased broad absorption background between 450 and $550 \mathrm{~nm}$ and at long wavelengths (>1000 $\mathrm{nm}$ ) seen in the doped TAPC film has been attributed to a higher refractive index because of the $\mathrm{MoO}_{3}$ clusters. ${ }^{41}$

The nature of the excited states and origin of the observed transitions in $\mathrm{TAPC}^{\bullet+}$ are elucidated via DFT and TD-DFT calculations. ${ }^{26}$ The orbital energy levels and optical transitions after cation formation (by removal of a $\beta$-electron) are schematically summarized in Figure $1 \mathrm{~d}$, and the respective orbitals are included in Figure S3. Note that in radical ions the energy levels differ for electrons of different spin $(\alpha$ and $\beta)$. The energy levels of TAPC $^{\bullet+}$ lie systematically below the corresponding ones of neutral TAPC but show a similar alignment. The higher-lying occupied levels (originating from the nearly degenerate HOMO and HOMO-1 of neutral TAPC) are close in energy, while there is a large gap with respect to the lower-lying occupied orbitals, which have a different distribution of nodal planes. To illustrate the optical transitions, the natural transition orbitals (NTOs), which represent the location of the electron and hole in the excited state, are shown in Figure S4.

The lowest excited state $\left(\mathrm{D}_{1}\right)$ of $\mathrm{TAPC}^{\bullet+}$ is populated by the transition of a $\beta$-electron from the highest occupied spinorbital (SOMO) to the singly unoccupied spin-orbital (SUMO) and occurs at very low energy $(\sim 0.4 \mathrm{eV})$, outside the spectral window shown in Figure 1c. The observed structured absorption band between 550 and $1000 \mathrm{~nm}(\sim 1.8-$ $2.2 \mathrm{eV}$ ) involves several $\beta$-electron transitions from deep-lying occupied orbitals to the SUMO and is collectively termed the $\mathrm{D}_{1}{ }^{\prime}$ state. Finally, the $\mathrm{D}_{0} \rightarrow \mathrm{D}_{2}$ transition at $350-400 \mathrm{~nm}$ $(\sim 3.2 \mathrm{eV})$ involves excitation of both $\alpha$ - and $\beta$-electrons predominantly from the higher-lying occupied spin-orbitals to the unoccupied orbitals and thus occurs across the bandgap of neutral TAPC.

We note that because of the peculiar electronic structure of TAPC $^{\bullet+}$, the lowest $\mathrm{D}_{0}-\mathrm{D}_{1}$ gap $(\sim 0.4 \mathrm{eV})$ is much smaller than the gaps between the higher excited states $\left(D_{1}-D_{1}{ }^{\prime}\right.$ and $\left.\mathrm{D}_{1}{ }^{\prime}-\mathrm{D}_{2},>1 \mathrm{eV}\right)$. Our previous observation of $\mathrm{D}_{1}{ }^{\prime} \rightarrow \mathrm{D}_{0}$ photoluminescence implies a violation of Kasha's rule that agrees with this energy gap alignment. ${ }^{42}$ This is also consistent with our finding below that nonradiative decay from the $\mathrm{D}_{1}{ }^{\prime}$ and $\mathrm{D}_{2}$ states is slower than internal conversion (IC) from the $\mathrm{D}_{1}$ state to the ground state, allowing charge transfer processes from the relatively long-lived higher excited states. 

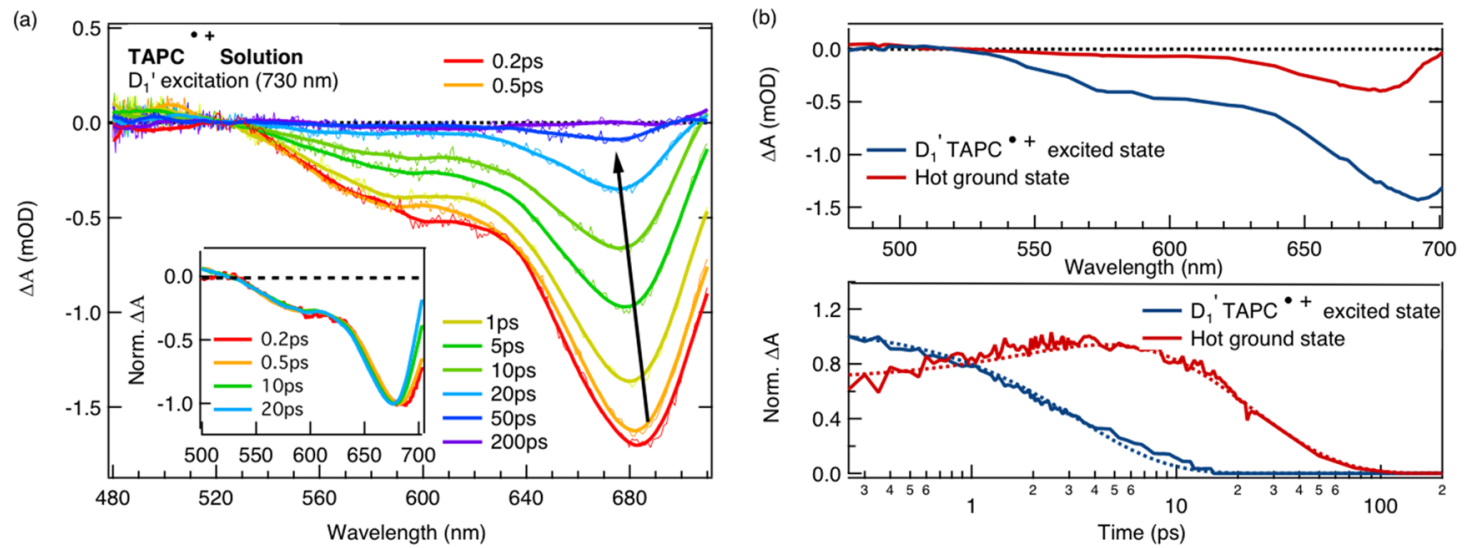

Figure 2. $\mathrm{D}_{1}{ }^{\prime}$ photoexcitation of TAPC ${ }^{\bullet+}$ solution. (a) Transient absorption spectra of TAPC ${ }^{\bullet+}$ solution in $\mathrm{H}_{2} \mathrm{SO}_{4}$ with $730 \mathrm{~nm}$ excitation pulses for an absorbed photon fluence of $\sim 2.5 \times 10^{13}$ photons $/ \mathrm{cm}^{2}$ at selected time delays after photoexcitation. In the inset, the normalized transient absorption spectra with $600 \mathrm{~nm}$ excitation are displayed. (b) Component spectra (top) and time-dependent normalized concentrations (bottom) of TAPC $^{\bullet+}$ solution obtained from soft-modeling MCR-ALS analysis of the transient absorption data with $730 \mathrm{~nm}$ excitation. Dotted lines show multiexponential fits of the resolved dynamics.

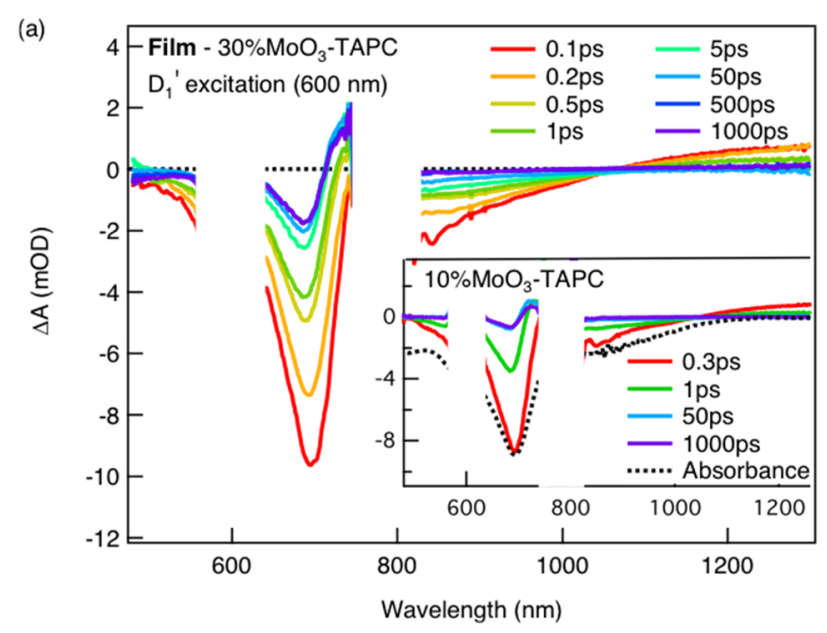

(c)

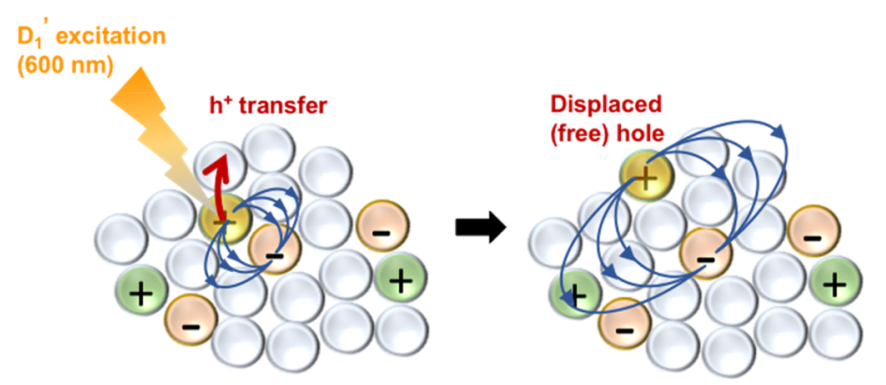

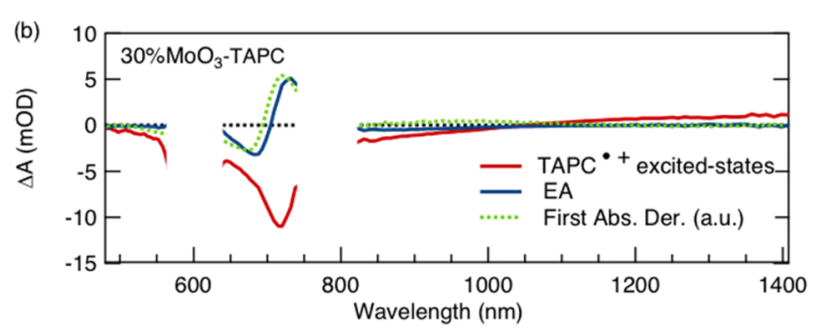

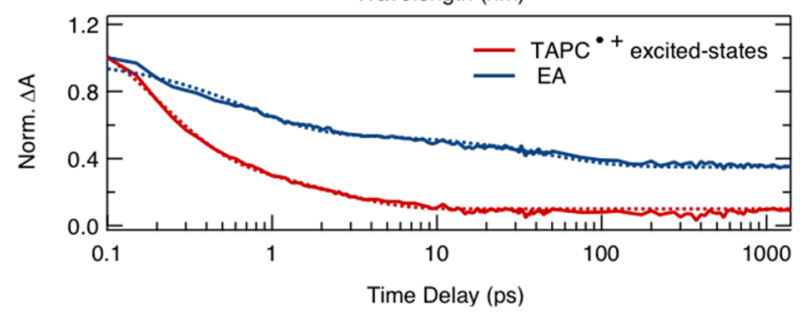

(d)

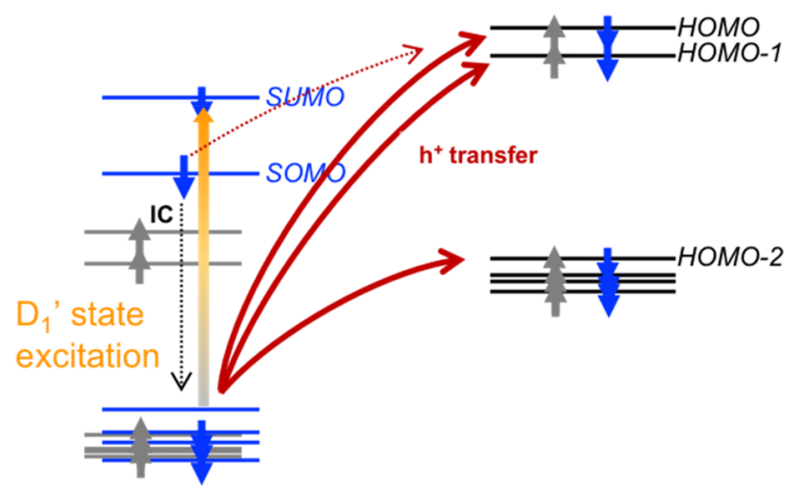

Figure 3. $\mathrm{D}_{1}{ }^{\prime}$ photoexcitation of doped TAPC films. (a) Transient absorption spectra of doped $30 \% \mathrm{MoO}_{3}-\mathrm{TAPC}$ and $10 \% \mathrm{MoO}_{3}-\mathrm{TAPC}$ (inset) films with $600 \mathrm{~nm}$ excitation pulses for an absorbed photon fluence of $\sim 4 \times 10^{14}$ photons $/ \mathrm{cm}^{2}$ at selected time delays after photoexcitation. (b) Component spectra (top) and time-dependent normalized concentrations (bottom) of doped $30 \% \mathrm{MoO}_{3}-\mathrm{TAPC}$ film obtained from softmodeling MCR-ALS analysis of the transient absorption data. Dotted lines show multiexponential fits of the resolved dynamics. (c) Schematic representation of doped $\mathrm{MoO}_{3}$-TAPC films, including the proposed photophysical processes upon $\mathrm{D}_{1}{ }^{\prime}$ excitation: Hole transfer from TAPC ${ }^{\bullet+}$ to a neutral molecule results in a new cation further away from the negatively charged $\mathrm{MoO}_{3}$ cluster, giving rise to long-lived electro-absorption (EA) signal due to an increased electric field. (d) Dominant electron configurations for $\mathrm{TAPC}^{\circ+}$ in the $\mathrm{D}_{1}{ }^{\prime}$ excited state and neutral TAPC in the ground state (according to DFT calculations), with different hole transfer pathways from the $\mathrm{D}_{1}{ }^{\prime}$ state generating a new cation either in the ground state or in one of the excited states (red solid arrows). The dotted arrows represent relaxation (by internal conversion (IC)) to the lowest $\mathrm{D}_{1}$ state followed by hole transfer. 
Table 1. Rise and Decay Time Constants and Their Normalized Respective Amplitudes (in Parentheses) Obtained from Multiexponential Fitting of the MCR-ALS Concentration Profiles of the TA Data in $30 \% \mathrm{MoO}_{3}-$ TAPC Film (or Undoped TAPC Film) upon $\mathrm{D}_{1}{ }^{\prime}, \mathrm{D}_{2}$, and $\mathrm{S}_{1}$ Excitation

\begin{tabular}{|c|c|c|c|c|c|}
\hline excitation & probed species & $\tau_{1}(\mathrm{ps})$ & $\tau_{2}(\mathrm{ps})$ & $\tau_{3}(\mathrm{ps})$ & offset \\
\hline \multirow[t]{2}{*}{$\mathrm{D}_{1}^{\prime}$ state $(600 \mathrm{~nm})$} & $\mathrm{TAPC}^{\bullet+}$ excited states & $0.2(0.7)$ & $2(0.2)$ & & $>1 \mathrm{~ns}(0.1)$ \\
\hline & EA & $0.7(0.4)$ & $47(0.2)$ & & $>1 \mathrm{~ns}(0.4)$ \\
\hline \multirow[t]{3}{*}{$\mathrm{D}_{2}$ state $(400 \mathrm{~nm})$} & $\mathrm{TAPC}^{\bullet+}$ excited states & $0.2(0.5)$ & $2(0.2)$ & $60(0.15)$ & $>1 \mathrm{~ns}(0.15)$ \\
\hline & $\mathrm{S}_{1}$ excited state & 0.1 (rise) & $11(0.5)$ & $190(0.5)$ & \\
\hline & EA & 0.1 (rise) & $0.4(0.4)$ & $100(0.2)$ & $>1 \mathrm{~ns}(0.4)$ \\
\hline \multirow{3}{*}{$\mathrm{S}_{1}$ and $\mathrm{D}_{2}$ states $(340 \mathrm{~nm})$} & $\mathrm{TAPC}^{\bullet+}$ excited states & $0.2(0.5)$ & $2(0.15)$ & $60(0.2)$ & $>1 \mathrm{~ns}(0.15)$ \\
\hline & $S_{1}$ excited state & 0.14 (rise) & $11(0.4)$ & $190(0.5)$ & $>1 \mathrm{~ns}(0.1)$ \\
\hline & EA & 0.14 (rise) & 10 (rise) & $100(0.3)$ & $>1 \mathrm{~ns}(0.7)$ \\
\hline $\mathrm{S}_{1}$ state $(340 \mathrm{~nm}$, undoped $)$ & $\mathrm{S}_{1}$ excited state & & $30(0.3)$ & $190(0.4)$ & $>1 \mathrm{~ns}(0.3)$ \\
\hline
\end{tabular}

As seen in Figure 1c, the ratio of the cation-related bands (transitions to the $\mathrm{D}_{1}{ }^{\prime}$ and $\mathrm{D}_{2}$ states) compared to the $\mathrm{S}_{1}$ band of neutral TAPC scales with $\mathrm{MoO}_{3}$ concentration, indicating a higher TAPC $^{\bullet+}$ concentration in the $30 \% \mathrm{MoO}_{3}$-TAPC film. To estimate the density of $\mathrm{TAPC}^{\bullet+}$ in the films, we have used the extinction coefficient found in electrochemically oxidized TAPC, ${ }^{41}$ the amplitude of the $\mathrm{D}_{1}{ }^{\prime}$ absorption band, and the film thickness $\left(d=220 \mathrm{~nm}\right.$ in $30 \% \mathrm{MoO}_{3}-$ TAPC and $750 \mathrm{~nm}$ in $\left.10 \% \mathrm{MoO}_{3}-\mathrm{TAPC}\right)$. We find that the density of cations is $\sim 8.7 \times 10^{19} \mathrm{~cm}^{-3}$ in $30 \% \mathrm{MoO}_{3}-$ TAPC and $\sim 2.3 \times 10^{19} \mathrm{~cm}^{-3}$ in $10 \% \mathrm{MoO}_{3}$-TAPC films. Given the molecular density of TAPC $\left(\sim 10^{21} \mathrm{~cm}^{-3}\right.$ based on a mass density of $\left.1.118 \mathrm{~g} / \mathrm{cm}^{3}\right)$, we estimate that $\sim 8 \%$ of all TAPC molecules are ionized in $30 \% \mathrm{MoO}_{3}-$ TAPC and $2 \%$ in $10 \% \mathrm{MoO}_{3}-$ TAPC films. The ionization fraction is lower than the doping concentration due to clustering of transition metal oxides during vacuum evaporation, which decreases their interface with the host material and causes their inhomogeneous distribution. ${ }^{43,44} \mathrm{We}$ conclude that in the doped films the $\mathrm{TAPC}^{\bullet+}$ cations are surrounded mainly by neutral TAPC molecules, as schematically indicated in Figure $1 \mathrm{~b}$.

First, we discuss the TA results when exciting the $\mathrm{D}_{0} \rightarrow \mathrm{D}_{1}{ }^{\prime}$ transitions at 730 or $600 \mathrm{~nm}$ in dissolved TAPC $^{\bullet+}$ (Figure 2 and Figure S5). Similar results were obtained at the two excitation wavelengths. Because less pump scattering is observed in the probed spectral window with $730 \mathrm{~nm}$ excitation, this data was used for further analysis. In the early TA spectra, the broad negative band is attributed to the ground-state bleaching of the $\mathrm{D}_{0} \rightarrow \mathrm{D}_{1}{ }^{\prime}$ transitions $\left(\mathrm{D}_{1}{ }^{\prime} \mathrm{GSB}\right)$ that overlaps with $\mathrm{D}_{1}{ }^{\prime}$ stimulated emission $\left(\mathrm{D}_{1}{ }^{\prime} \mathrm{SE}\right)$ on the red side of the band. To distinguish between spectral components of different origin, we have analyzed our data using softmodeling multivariate curve resolution analysis (MCRALS).$^{45-48}$ Following this procedure, we deconvolute the TA spectra into spectra associated with the photogenerated species and their corresponding time-dependent concentrations (top and bottom of Figure $2 b$, respectively). We resolve two spectral components with quite similar negative features, having a small spectral shift between them and showing different dynamics. The faster-decaying component (blue solid line) contains contributions from overlapping $\mathrm{D}_{1}{ }^{\prime} \mathrm{GSB}$ and $\mathrm{D}_{1}{ }^{\prime} \mathrm{SE}$ signatures and is assigned to the directly excited $\mathrm{D}_{1}{ }^{\prime}$ state of $\mathrm{TAPC}^{\bullet+}$. We find that it decays monoexponentially with a time constant of $\sim 3.3$ ps. The second component (red solid line) contains only the $\mathrm{D}_{1}{ }^{\prime} \mathrm{GSB}$ and no SE, so that it appears to be blue-shifted. It rises with a time constant of $\sim 3$ ps similar to the depopulation of the $\mathrm{D}_{1}{ }^{\prime}$ excited state and decays within tens of picoseconds ( $\sim 23 \mathrm{ps})$. This component could in principle represent the lowest $\mathrm{D}_{1}$ excited state, but this is unlikely since the $\mathrm{D}_{1}$ state lies only $0.4 \mathrm{eV}$ above the ground state and is therefore expected to have an extremely short lifetime. We therefore assign the signature to a vibrationally hot electronic ground state (HGS). Ultrafast decay of the first excited state and HGS signatures were also observed for cationic Wurster's salts and $\mathrm{C}_{60}$ radical anions in solution. ${ }^{19,49}$ The short excited-state lifetime of radical ions in small molecules $^{17,19,49-52}$ and conjugated polymers ${ }^{7}$ is a consequence of their low-lying excited states (energy gap law) ${ }^{53}$ and/or the presence of conical intersections between their ground and excited states. 54

In addition, TA measurements were performed on $\mathrm{MoO}_{3}$ doped TAPC films, where increased intermolecular interactions due to close packing of molecules are expected. The $\mathrm{TAPC}^{\bullet+}$ cations are now surrounded by neutral TAPC molecules, which are less abundant in the TAPC $^{\bullet+}$ solution and only accessible by diffusion. We have used different excitation wavelengths (vertical dotted lines in Figure 1c) to resonantly excite the $\mathrm{D}_{1}{ }^{\prime}(600 \mathrm{~nm}), \mathrm{D}_{2}(400 \mathrm{~nm})$, and neutral TAPC $S_{1}$ state $(340 \mathrm{~nm})$ in the films. The TA spectra for doped $30 \% \mathrm{MoO}_{3}-\mathrm{TAPC}$ and $10 \% \mathrm{MoO}_{3}$-TAPC (inset) films when exciting the $\mathrm{D}_{0} \rightarrow \mathrm{D}_{1}{ }^{\prime}$ transitions of $\mathrm{TAPC}^{\bullet+}$ at an absorbed photon fluence of $\sim 4 \times 10^{14}$ photons $/ \mathrm{cm}^{2}$ are shown in Figure 3a. In the early TA spectra, the negative signal between 500 and $1000 \mathrm{~nm}$ is related to the $\mathrm{D}_{1}^{\prime}$ GSB and mirrors the corresponding absorption band (see the inset). The $\mathrm{D}_{1}{ }^{\prime}$ SE band is not clearly visible, since it is either too weak in the solid state or masked by the noise around $800 \mathrm{~nm}$ from the fundamental laser beam. A broad positive photoinduced absorption band at near-infrared (NIR) wavelengths $(>1000 \mathrm{~nm})$ is assigned to excited-state absorption of the cation $\left(\mathrm{TAPC}^{\bullet+}-\mathrm{ESA}\right)$. After about $1 \mathrm{ps}$, an oscillatory feature becomes evident near $700 \mathrm{~nm}$, which is clearly distinct from the signature of the HGS seen in solution and persists longer than the $\sim 1$ ns experimental window.

Spectral components and their respective dynamics from MCR-ALS analysis of the $30 \% \quad \mathrm{MoO}_{3}-\mathrm{TAPC}$ film are displayed in Figure $3 \mathrm{~b}$. The first spectral component (red line) contains the $\mathrm{D}_{1}{ }^{\prime}$ GSB and $\mathrm{TAPC}^{\bullet+}-\mathrm{ESA}$ bands and is thus assigned to the excited states of $\mathrm{TAPC}^{\bullet+}$. We note that it is difficult to distinguish the $\mathrm{D}_{1}$ and $\mathrm{D}_{1}{ }^{\prime} \mathrm{TAPC}^{\bullet+}$ excited states from their TA signature because they both display the same GSB, and both are separated from the next higher-lying state $\left(\mathrm{D}_{1}{ }^{\prime}\right.$ and $\mathrm{D}_{2}$, respectively) by about $1 \mathrm{eV}$, so that absorption in the NIR occurs. However, for the reasons discussed above in solution, we expect the $\mathrm{D}_{1}{ }^{\prime}$ state to dominate since the lowlying $\mathrm{D}_{1}$ state should undergo ultrafast nonradiative decay (by 

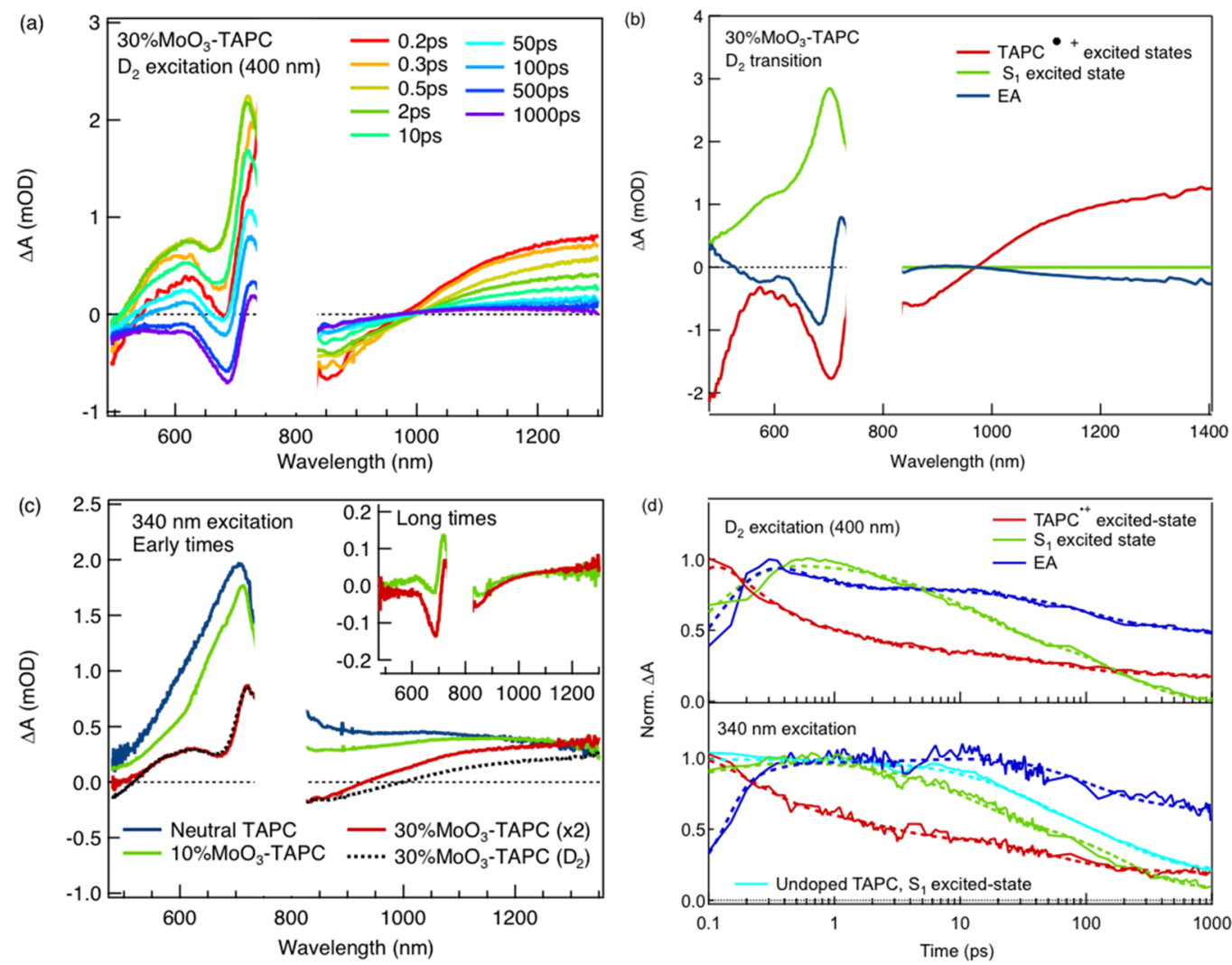

Figure 4. $\mathrm{D}_{2}$ and $\mathrm{S}_{1}$ photoexcitation of doped TAPC films. (a) Transient absorption spectra of doped $30 \% \mathrm{MoO}_{3}-\mathrm{TAPC}$ film with $400 \mathrm{~nm}$ excitation pulses $\left(D_{2}\right.$ excitation) at an absorbed photon fluence of $\sim 3.5 \times 10^{14}$ photons $/ \mathrm{cm}^{2}$ at selected time delays after photoexcitation. (b) Component spectra of doped $30 \% \mathrm{MoO}_{3}$-TAPC film obtained from soft-modeling MCR-ALS analysis upon $\mathrm{D}_{2}$ pumping. (c) Transient absorption spectra of undoped TAPC, doped $10 \% \mathrm{MoO}_{3}-$ TAPC and $30 \% \mathrm{MoO}_{3}-\mathrm{TAPC}$ films excited after excitation at $340 \mathrm{~nm}$, scaled to an absorbed photon fluence of $\sim 5 \times 10^{13}$ photons $/ \mathrm{cm}^{2}$, at early times $(0.5 \mathrm{ps})$ and long times $(1 \mathrm{~ns}$, shown as inset). The scaled transient absorption spectrum of $30 \% \mathrm{MoO}_{3}-$ TAPC excited at $400 \mathrm{~nm}$ (to the $\mathrm{D}_{2}$ state, at $0.5 \mathrm{ps}$ ) is also included. (d) Time-dependent normalized concentrations of doped $30 \% \mathrm{MoO}_{3}$-TAPC film obtained from soft-modeling MCR-ALS analysis upon $\mathrm{D}_{2}$ (top) and $340 \mathrm{~nm}$ (bottom) pumping. Dotted lines show multiexponential fits of the resolved dynamics. The dynamics of the $S_{1}$ state from the undoped TAPC film is also shown.

IC) to the ground state. Most of the $\mathrm{TAPC}^{\bullet+}$ excited-state signatures decay with time constants of $\sim 200 \mathrm{fs}(70 \%)$ and $\sim 2$ ps $(20 \%)$, though a $10 \%$ offset remains at long times (Figure $3 \mathrm{~b}$, lower panel, and Table 1). Such fast excited-state decay agrees well with the weak PL signal from $\mathrm{MoO}_{3}$-doped films (quantum yield of $\sim 10^{-4}$ ). ${ }^{42}$ It is shorter in the films compared to solution, and the HGS signature is absent, which we attribute to faster vibrational relaxation in the solid state due to the larger number of dissipation channels and to quenching of the excitation by neutral TAPC molecules (see below). We note that given the short-lived excited states, annihilation processes are absent over a large range of pump fluences and the TA dynamics are independent of excitation density (Figure S6). Interestingly, the second spectral component (blue line) has a similar shape as the first derivative of the $\mathrm{D}_{1}{ }^{\prime}$ absorption bands of $\mathrm{TAPC}^{\circ+}$. Its presence only in the solid state indicates that it arises from the close packing of molecules. A similar derivative-like feature due to electro-absorption (EA) is often present in the TA spectra of organic semiconductors and originates from local electric fields induced by photogenerated charges. ${ }^{55-59}$ For photoexcited donor:acceptor blends in organic solar cells, the evolution of this EA signal has been related to the spatial separation of Coulombically bound charge pairs formed after exciton dissociation at an interface. ${ }^{40,55-57}$ We therefore suggest that the first derivative component seen in the $\mathrm{MoO}_{3}$-doped TAPC films is caused by excited holes that hop away from their $\mathrm{MoO}_{3}$ counterion, perturbing the equilibrium electrostatic environment experienced by other (ground state) cations.

To explain how photoexcitation of $\mathrm{TAPC}^{\bullet+}$ can lead to such an effect, we consider the composition of doped TAPC films (Figure 3c) and the calculated energy level diagram (Figure $3 \mathrm{~d})$. As mentioned above, most of the $\mathrm{TAPC}^{\bullet+}$ cations are Coulombically bound to the negatively charged dopant clusters but are surrounded by neutral TAPC molecules because of the low doping concentration. According to the energetic alignment between the orbitals and the proximity of molecules in the films, HT from photoexcited $\mathrm{TAPC}^{\bullet+}$ to an adjacent neutral TAPC molecule is possible. The initially excited TAPC $^{\bullet+}$ thus becomes a neutral molecule while the second molecule becomes a new cation, corresponding to movement of the excited hole. This is favored by excess excitation energy compared to ground-state hole transport. Excited-state charge migration has also been reported in dyads of radical ions with their neutral counterparts and has been proposed to model charge transport in organic solids. ${ }^{12,60}$ Importantly, the new cation in the doped TAPC films may be located further away from the negatively charged $\mathrm{MoO}_{3}$ cluster and thus less Coulombically bound to it. The magnitude of the electric dipole formed with the $\mathrm{MoO}_{3}$ anion thus increases with respect to the equilibrium case, giving rise to the observed EA signature, since the electric field experienced by other nearby 
Scheme 1. Involved Orbital Energy Levels and Electron Configurations from DFT Calculations with Excitation of (a) the $\mathrm{D}_{2}$ State of TAPC $^{\bullet+}$ and (b) the $S_{1}$ State of TAPC in Doped Films Including the Charge Transfer Processes between Cations and Neutral TAPC Molecules (Only $\beta$-Electron Transitions Are Shown for Simplicity); (c) Schematic Representation of Doped $\mathrm{MoO}_{3}$-TAPC Films, Including the Proposed Photophysical Processes upon $\mathrm{D}_{2}$ and $S_{1}$ Photoexcitation, Showing the Migration of the Hole Carrier

(a) $D_{2}$ state excitation

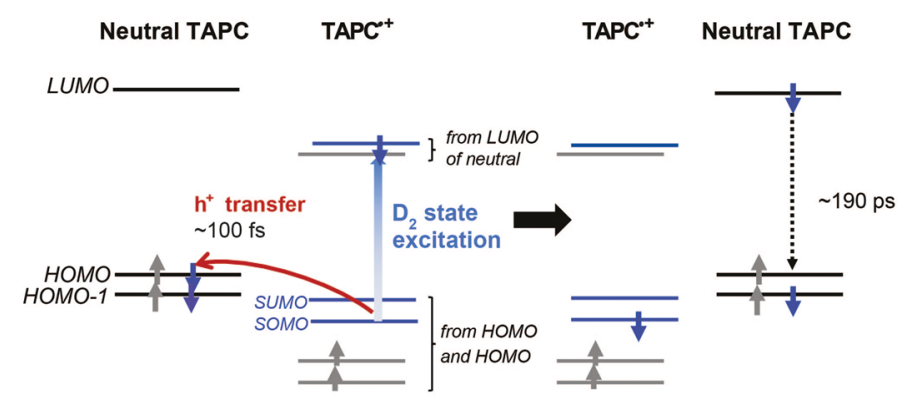

(c) $\mathrm{S}_{1}$ state excitation

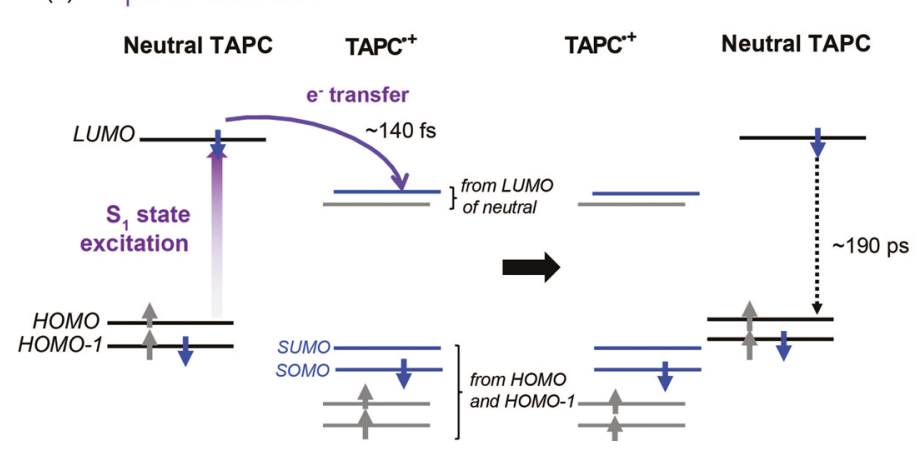

(b)

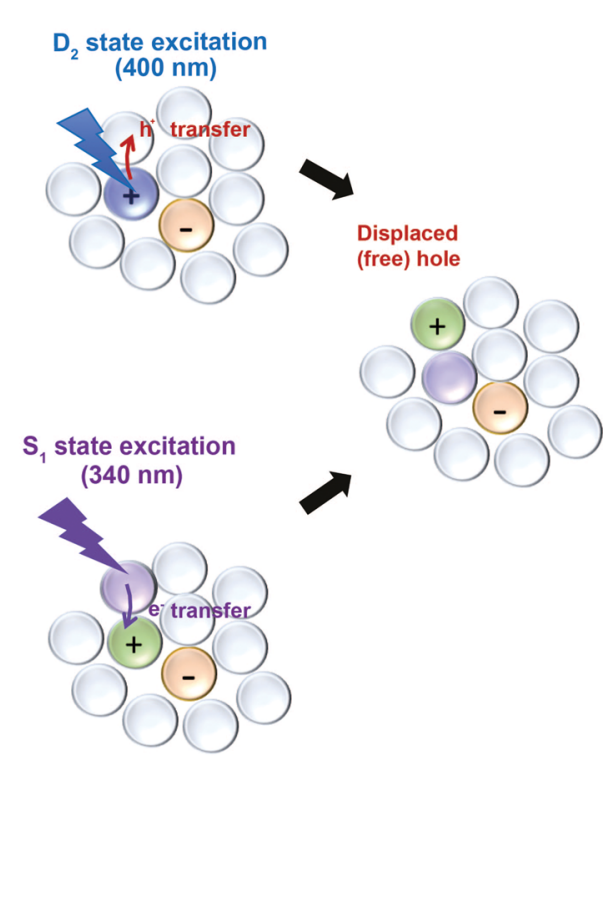

$\mathrm{TAPC}^{\bullet+}$ cations is perturbed and shifts their $\mathrm{D}_{1}{ }^{\prime}$ transition (Figure 3c). The first derivative line shape indicates that the shift is related to a change in polarizability between the ground and excited states of TAPC ${ }^{\bullet+59}$ The EA signal observed in the $10 \% \mathrm{MoO}_{3}$-TAPC film (inset of Figure 3a) is smaller relative to the initial $\mathrm{D}_{1}{ }^{\prime} \mathrm{GSB}$ (which reflects the initial number of excitations), as fewer $\mathrm{TAPC}^{\bullet+}$ cations experience the electric field change at the lower doping concentration.

Judging from the energy levels in Figure $3 \mathrm{~d}$, we cannot exclude that some HT to neutral TAPC molecules occurs from the lowest-lying $\mathrm{D}_{1}$ excited state of $\mathrm{TAPC}^{\bullet+}$, populated by relaxation from the photoexcited $\mathrm{D}_{1}{ }^{\prime}$ state. However, because of the unusual energy gap alignment, HT predominantly occurs from the higher $\mathrm{D}_{1}{ }^{\prime}$ state (which lives longer than the $D_{1}$ state), generating the $D_{1}{ }^{\prime}, D_{1}$, or ground state of the newly formed cation. The latter two pathways cause the observed rapid deactivation $(\sim 200 \mathrm{fs}$, Table 1$)$ of the $\mathrm{TAPC}^{\bullet+}$ excitation. HT to lower-lying occupied levels of the initially neutral molecule maintains the $\mathrm{D}_{1}{ }^{\prime}$ excitation, contributing to the intermediate $(\sim 2 \mathrm{ps})$ time constant seen in the TA data, which is similar to the intrinsic $\mathrm{D}_{1}^{\prime}$ lifetime observed in solution. Interestingly, a small fraction $(10 \%)$ of $\mathrm{TAPC}^{\bullet+}$ remains in the excited state at long times $(>1 \mathrm{~ns})$, in spite of the $\sim 2-3$ ps intrinsic excited-state lifetime of the cations. We suggest that $\mathrm{TAPC}^{\bullet+}$ excited states are gradually repopulated by back-transfer from vibrationally hot neutral TAPC molecules (formed by HT from photoexcited TAPC $^{\bullet+}$ ) to adjacent radical cations. ${ }^{11,19}$ An alternative cause for a persisting $\mathrm{D}_{1}{ }^{\prime} \mathrm{GSB}$ could be excited-state $\mathrm{HT}$ to $\mathrm{MoO}_{3}$ anions, neutralizing the charges. This cannot explain the long-lived $\mathrm{TAPC}^{\bullet+}-\mathrm{ESA}$, which becomes more pronounced at higher pump energies (see below).

Finally, considering the dynamics of the EA signature (bottom of Figure $3 \mathrm{~b}$ ), the maximum EA signal is observed promptly after photoexcitation, confirming that the HT process is ultrafast $(<100 \mathrm{fs})$ and occurs largely within the time resolution of our experiment. This allows the process to compete with the intrinsic deactivation of the $\mathrm{D}_{1}^{\prime}$ excited state ( $\sim 2-3 \mathrm{ps})$. Then, the EA signal partly decays with time constants of $\sim 0.7$ ps $(40 \%)$ and $\sim 47$ ps (20\%) (Table 1 ). Because the hole carriers can continue to hop within the film (by fast excited-state or slower ground-state charge transfer to adjacent neutral sites), they can become trapped again at an ionized $\mathrm{MoO}_{3}$ site, causing the decay of the EA signature. There is nevertheless a significant long-lived offset in the EA (40\%), indicating that a large fraction of $\mathrm{TAPC}^{\bullet+}$ cations remains away from their equilibrium position at long time delays (>1 ns), presumably because some of them have completely separated from their counterion. Thus, for doped TAPC films, one or more excited-state hops (before TAPC ${ }^{\bullet+}$ returns to the ground state) facilitate hole transport and separation from the ionized dopant, leading to the photocurrent in devices that we previously reported in ref 16 .

We also performed TA measurements when selectively pumping the $\mathrm{D}_{0} \rightarrow \mathrm{D}_{2}$ transition of the $\mathrm{TAPC}^{\bullet+}$ cations in $30 \% \mathrm{MoO}_{3}$-TAPC films at $400 \mathrm{~nm}$ (Figure 4a, absorbed photon fluence $\sim 3.5 \times 10^{14} \mathrm{~cm}^{-2}$ ). From early times on, the TA spectra differ significantly from those obtained with $\mathrm{D}_{1}{ }^{\prime}$ excitation, having an additional positive band overlapping with the signatures of the $\mathrm{TAPC}^{\circ+}$ excited states $\left(\mathrm{D}_{1}{ }^{\prime} \mathrm{GSB}\right.$, $\left.\mathrm{TAPC}^{\circ+}-\mathrm{ESA}\right)$ and the oscillatory EA signal. The three 
spectral components are well resolved via MCR-ALS analysis (Figure $4 \mathrm{~b}$ ), allowing us to assign the additional positive band to $S_{1}$ excited-state absorption $\left(S_{1}\right.$-ESA) of neutral TAPC molecules based on its similarity to the spectrum obtained when directly exciting an undoped TAPC film at $340 \mathrm{~nm}$ (Figure 4c). We note that no detectable TA signal is obtained when the undoped film is pumped at $400 \mathrm{~nm}$, excluding direct excitation of neutral TAPC molecules at this wavelength as the origin of the observed $\mathrm{S}_{1}$-ESA band in the doped $30 \% \mathrm{MoO}_{3}-$ TAPC film. Similar TA spectra are observed at lower doping concentration in the $10 \% \mathrm{MoO}_{3}-\mathrm{TAPC}$ film (Figure S7). We propose that upon excitation of the $\mathrm{D}_{0} \rightarrow \mathrm{D}_{2}$ transition at 400 $\mathrm{nm}$ the $\mathrm{S}_{1}$ state is populated by HT from the excited cation to an adjacent neutral TAPC molecule, involving predominantly the higher-lying occupied levels rather than the deeper-lying occupied orbitals (Scheme 1a). Unlike with $\mathrm{D}_{1}{ }^{\prime}$ excitation, the initially photoexcited $\mathrm{TAPC}^{\bullet+}$ is converted to a neutral TAPC molecule in the excited state (responsible for the $S_{1}$-ESA signature), while the new $\operatorname{TAPC}^{\bullet+}$ cation is formed in the ground state. This new cation is spatially separated from the $\mathrm{MoO}_{3}$ anion (Scheme $1 \mathrm{~b}$ ) and causes the EA signature as observed with $\mathrm{D}_{1}{ }^{\prime}$ excitation. Again, migration of the excited hole perturbs the electrostatic equilibrium in the film and facilitates the generation of free carriers, as indicated by the important (40\%) long-lived offset in the EA (Table 1).

A significant fraction of the $S_{1}$ state and EA appears within the time resolution of the experiment, with a further rise of the two signals occurring with $\sim 0.1$ ps (Figure $4 \mathrm{~d}$ and Table 1 ). The slower rise of the EA compared to that seen with excitation of the $D_{1}{ }^{\prime}$ state $(<100 \mathrm{fs}$, Figure $3 \mathrm{~b})$ points to slower HT from the $D_{2}$ state. This is related to a lower energetic driving force, ${ }^{12,14,61,62}$ given the smaller energy offset between the higher-lying occupied levels of $\mathrm{TAPC}^{\bullet+}$ and neutral TAPC, with respect to the deeper-lying orbitals involved in HT from the $\mathrm{D}_{1}{ }^{\prime}$ state. Nevertheless, HT is still faster than IC from the $\mathrm{D}_{2}$ to the $\mathrm{D}_{1}{ }^{\prime}$ excited state, which is again slowed by a large energy gap and transition to a significantly different electronic configuration. The slightly different shape of the MCR-ALS component representing the $\mathrm{TAPC}^{\bullet+}$ excited states (enhanced TAPC $^{\bullet+}$-ESA) might show the contribution of the $\mathrm{D}_{2}$ state (Figure $4 \mathrm{~b}$ ). Part of the $\mathrm{D}_{2}$ population still relaxes to the $\mathrm{D}_{1}{ }^{\prime}$ state, as emission from the $\mathrm{D}_{1}{ }^{\prime}$ state when exciting the $\mathrm{D}_{0} \rightarrow$ $\mathrm{D}_{2}$ transition has been reported. ${ }^{42}$ From there, similar processes occur as with direct $\mathrm{D}_{1}{ }^{\prime}$ excitation. The signature of the $\mathrm{TAPC}^{\bullet+}$ excited states with $400 \mathrm{~nm}$ excitation decays again with time constants of $0.2 \mathrm{ps}(50 \%)$ and 2 ps (\%) as well as longer components of 60 ps (15\%) and an offset (15\%) (Table 1), which are enhanced compared to direct $\mathrm{D}_{1}{ }^{\prime}$ excitation at $600 \mathrm{~nm}$. As explained above, the long-lived $\mathrm{TAPC}^{\bullet+}$ excited states are populated by back-transfer from hot neutral TAPC molecules (having more excess energy with 400 $\mathrm{nm}$ excitation). Additionally, there can be excitation energy transfer (EET) from the $S_{1}$ excited state of TAPC molecules to TAPC $^{\bullet+}$, as discussed below.

When the undoped TAPC film is excited at $340 \mathrm{~nm}$, the characteristic $S_{1}$-ESA appears instantaneously within the time resolution of the experiment and decays with time constants of $\sim 30$ ps (30\%), $\sim 190$ ps (40\%), and an offset (30\%) (Figure $4 \mathrm{~d}$ and Figure S8). In the doped films, both the TAPC ${ }^{\bullet+}$ cations $\left(D_{0} \rightarrow D_{2}\right.$ transition $)$ and neutral molecules $\left(S_{0} \rightarrow S_{1}\right.$ transition) are photoexcited at $340 \mathrm{~nm}$, but considering the low cation concentration, the TA features from neutral molecules are expected to dominate. This is indeed the case in the less doped $10 \% \mathrm{MoO}_{3}$-TAPC film (Figure 4c), where the early TA spectrum resembles that of undoped TAPC. In contrast, for the $30 \% \mathrm{MoO}_{3}-\mathrm{TAPC}$ film excited at $340 \mathrm{~nm}$, similar TA spectra and MCR-ALS dynamics are obtained as with selective $\mathrm{D}_{2}$ excitation at $400 \mathrm{~nm}$ (Figure $4 c, d$ and Figure S9). At both doping levels, the EA signature representative of holes separating from their $\mathrm{MoO}_{3}$ counterions is evident at late times (inset of Figure 4c). To explain this, we consider the energy levels of neutral TAPC in the $S_{1}$ excited state and of $\mathrm{TAPC}^{\bullet+}$ in the ground state (Scheme 1c), which reveal that ET between their LUMO levels is energetically feasible. The initially photoexcited neutral TAPC molecule converts to $\mathrm{TAPC}^{\bullet+}$ in the ground state, while the initial cation becomes a neutral TAPC in the $S_{1}$ excited state, effectively resulting in the migration of the hole and neutral excitation in opposite directions and contributing to movement of the hole relative to its counterion that leads to the EA signature. Alternatively, given the overlap of the neutral TAPC emission and TAPC ${ }^{\bullet+}$ $\mathrm{D}_{2}$ absorption, EET can occur, whereby the excitation of neutral TAPC is quenched while excited-state $\mathrm{TAPC}^{\bullet+}$ is generated, facilitating subsequent hole hopping. This mechanism agrees with the previously observed reduction of $S_{1}$ emission in the doped films. ${ }^{42}$ Here, we see only a slight shortening of the $\mathrm{S}_{1}$ lifetime in the doped $30 \% \mathrm{MoO}_{3}$-TAPC compared to the undoped TAPC film excited at $340 \mathrm{~nm}$ (Figure $4 \mathrm{~d}$ and Table 1). Therefore, most EET likely occurs faster than the experimental time resolution, populating the $\mathrm{D}_{2}$ state of $\mathrm{TAPC}^{\bullet+}$ and explaining the similarity with the TA data at $400 \mathrm{~nm}$. At long times, the enhanced repopulation of the TAPC $^{\bullet+}$ excited states is shown by their higher offset $(15 \%)$ with $340 / 400 \mathrm{~nm}$ excitation compared to $600 \mathrm{~nm}$ excitation (10\%), suggesting the occurrence of some slow EET as well (Table 1).

To summarize the processes that take place at high energy excitation $(340$ or $400 \mathrm{~nm})$ in the doped TAPC films: (i) If a neutral TAPC molecule that is in close vicinity to a TAPC ${ }^{\bullet+}$ cation is directly excited at $340 \mathrm{~nm}$ (more likely at higher doping level in the $30 \% \mathrm{MoO}_{3}-\mathrm{TAPC}$ film), ultrafast ET $(0.14 \mathrm{ps})$ and/or EET $(<0.1 \mathrm{ps})$ from the $\mathrm{S}_{1}$ excited state leads to the displacement of a ground-state hole (causing the EA signature) and/or the generation of an excited $\mathrm{TAPC}^{\circ+}$ cation, respectively (Scheme 1c). (ii) The $\mathrm{D}_{2}$ state of TAPC (directly excited at 340 and $400 \mathrm{~nm}$ or formed via EET from the $S_{1}$ state) undergoes HT to neutral TAPC molecules $(0.1 \mathrm{ps})$, promoting the migration of a hole that loses its excitation (EA signature) and the formation of an excited neutral TAPC molecule $\left(S_{1}\right.$ state) in the process (Scheme 1a). Here, the rise of the $S_{1}$ excited state is less pronounced with $340 \mathrm{~nm}$ than $400 \mathrm{~nm}$ pumping (Figure $4 \mathrm{~d}$ and Table 1) because there is also direct $S_{1}$ excitation. Alternatively, the $D_{2}$ state relaxes to the $\mathrm{D}_{1}{ }^{\prime}$ states, which also facilitates hole hopping. (iii) Given the long lifetime of the $S_{1}$ state ( $\left.\sim 190 \mathrm{ps}\right)$, the neutral TAPC excitons can migrate within the film, followed by ET and/or some EET when they encounter a TAPC $^{\bullet+}$ cation. The benefit of maintaining long-lived excitation via the $S_{1}$ state reflects in an additional slow rise (10 ps) and higher offset (0.7\%) of the EA signature (Table 1 ), showing delayed formation and an enhanced yield of free hole carriers that cause photoconductivity.

\section{CONCLUSION}

To conclude, we have used femtosecond transient absorption spectroscopy on a doped organic solid to provide detailed 
insight into how photoexcitation of different electronic transitions allows bound holes to overcome the Coulombic interaction with the ionized dopant. Excited-state hopping facilitates the transport of holes by driving their transfer to adjacent neutral molecules (which is otherwise thermally activated $)^{9}$ with excess excitation energy. This enhances the yield of long-lived free charges, which we identify via their characteristic electro-absorption signature, and is the origin of the photoconductivity observed in ref 16 . The effect relies on the coexistence of closely packed charged and neutral molecules in the films and on a favorable energetic alignment between them, which we have demonstrated via DFT calculations.

Because of the peculiar electronic structure of $\mathrm{TAPC}^{\bullet+}$, having larger energy gaps between higher-lying electronic states than between the ground state and first excited state in violation of Kasha's rule, the ultrafast hole transfer to neutral TAPC can take place from the higher excited states in competition with internal conversion. This leads to ultrafast back-and-forth shuffling of charges and excitation energy between cations and adjacent neutral molecules. While excitedstate hopping is typically limited by the very short intrinsic lifetime of excited $\mathrm{TAPC}^{\bullet+}(\sim 2-3 \mathrm{ps})$, populating the longlived excited state of neutral TAPC $(\sim 190 \mathrm{ps})$ maintains the excitation in the films, promoting hole transport over long times by electron and excitation energy transfer processes and thus increasing the yield of conductive hole carriers.

Generalizing our findings, we have observed similar photoinduced hole transfer between polaronic and neutral sites in a self-doped conjugated polymer, ${ }^{7}$ but this time the strong coupling between the sites along the chain allowed direct charge transfer transitions from the ground state. Moreover, bound charge pairs formed at donor:acceptor interfaces in organic solar cell blends can also be dissociated by light using pump-push spectroscopies. ${ }^{13-15}$ Our findings therefore not only provide new fundamental insights about the photophysics of radical ions in the solid state but also are generally relevant to understanding separation of bound charges, for example, in doped organic systems or organic solar cells.

\section{ASSOCIATED CONTENT}

\section{(s) Supporting Information}

The Supporting Information is available free of charge at https://pubs.acs.org/doi/10.1021/acs.jpcc.1c01990.

S1: results from DFT and TD-DFT calculations; S2: additional results from TA measurements (PDF)

\section{AUTHOR INFORMATION}

\section{Corresponding Author}

Natalie Banerji - Department of Chemistry and Biochemistry, University of Bern, 3012 Bern, Switzerland; orcid.org/ 0000-0001-9181-2642; Email: natalie.banerii@ dcb.unibe.ch

\section{Authors}

Demetra Tsokkou - Department of Chemistry and Biochemistry, University of Bern, 3012 Bern, Switzerland

Chiao-Yu Cheng - Department of Electrical Engineering, The Pennsylvania State University, University Park, Pennsylvania 16802, United States
Nina Krainova - Department of Electrical Engineering, The Pennsylvania State University, University Park, Pennsylvania 16802, United States

Sukrit Mukhopadhyay - The Dow Chemical Company, Midland, Michigan 48674, United States

Noel C. Giebink - Department of Electrical Engineering, The Pennsylvania State University, University Park, Pennsylvania 16802, United States; 다. orcid.org/0000-0002-3798-5830

Complete contact information is available at:

https://pubs.acs.org/10.1021/acs.jpcc.1c01990

\section{Notes}

The authors declare no competing financial interest.

\section{ACKNOWLEDGMENTS}

D.T., and N.B. thank the European Research Council (ERC) for supporting this research by a Starting Grant (No. 714586, OSIRIS) and acknowledge NCCR-MUST, a research instrument of the Swiss National Science Foundation, as well as the University of Bern. N.B. thanks the Swiss National Science Foundation for research support (Grant 200020_184819). Work by C.C., N.K., and N.C.G. was supported in part by NSF Grant DMR-1654077 and the U.S. Department of Energy, Office of Basic Energy Sciences under Award No. DESC0012365.

\section{ABBREVIATIONS}

TA, transient absorption; HT, hole transfer; EA, electroabsorption; $\mathrm{H}_{2} \mathrm{SO}_{4}$, sulfuric acid; ET, electron transfer; DFT, density functional theory; HOMO, highest occupied molecular orbital; LUMO, lowest unoccupied molecular orbital; NTO, natural transition orbital; SOMO, singly occupied molecular orbital; SUMO, singly unoccupied molecular orbital; IC, internal conversion; GSB, ground-state bleaching; SE, stimulated emission; MCR-ALS, multivariate curve resolution analysis; HGS, hot ground state; NIR, near-infrared; ESA, excited-state absorption; EET, excitation energy transfer.

\section{REFERENCES}

(1) Jacobs, I. E.; Moulé, A. J. Controlling Molecular doping in organic semiconductors. Adv. Mater. 2017, 29 (42), 1703063.

(2) Lüssem, B.; Riede, M.; Leo, K. Doping of organic semiconductors. Phys. Status Solidi A 2013, 210 (1), 9-43.

(3) Tietze, M. L.; Burtone, L.; Riede, M.; Lüssem, B.; Leo, K. Fermi level shift and doping efficiency in p-doped small molecule organic. Phys. Rev. B: Condens. Matter Mater. Phys. 2012, 86, 035320.

(4) Arkhipov, V. I.; Heremans, P.; Emelianova, E. V.; Bässler, H. Effect of doping on the density-of-states distribution and carrier hopping in disordered organic semiconductors. Phys. Rev. B: Condens. Matter Mater. Phys. 2005, 71 (4), 045214.

(5) Etemad, S.; Heeger, A. J. Polyacetylene, $(\mathrm{CH}) \mathrm{x}$ : The Prototype Conducting Polymer. Annu. Rev. Phys. Chem. 1982, 33 (1), 443-469.

(6) Basescu, N.; Liu, Z. X.; Moses, D.; Heeger, A. J.; Naarmann, H.; Theophilou, N. High electrical conductivity in doped polyacetylene. Nature 1987, 327 (6121), 403-405.

(7) Tsokkou, D.; Peterhans, L.; Cao, D. X.; Mai, C.-K.; Bazan, G. C.; Nguyen, T.-Q.; Banerji, N. Excited State Dynamics of a Self-doped conjugated polyelectrolyte. Adv. Funct. Mater. 2020, 30 (9), 1906148.

(8) Gaul, C.; Hutsch, S.; Schwarze, M.; Schellhammer, K. S.; Bussolotti, F.; Kera, S.; Cuniberti, G.; Leo, K.; Ortmann, F. Insight into doping efficiency of organic semiconductors from the analysis of the density of states in n-doped $\mathrm{C}_{60}$ and ZnPc. Nat. Mater. 2018, 17 (5), 439-444. 
(9) Schwarze, M.; Gaul, C.; Scholz, R.; Bussolotti, F.; Hofacker, A.; Schellhammer, K. S.; Nell, B.; Naab, B. D.; Bao, Z.; Spoltore, D.; et al. Molecular parameters responsible for thermally activated transport in doped organic semiconductors. Nat. Mater. 2019, 18 (3), 242-248.

(10) Yurash, B.; Cao, D. X.; Brus, V. V.; Leifert, D.; Wang, M.; Dixon, A.; Seifrid, M.; Mansour, A. E.; Lungwitz, D.; Liu, T.; et al. Towards understanding the doping mechanism of organic semiconductors by Lewis acids. Nat. Mater. 2019, 18 (12), 1327-1334.

(11) Christensen, J. A.; Phelan, B. T.; Chaudhuri, S.; Acharya, A.; Batista, V. S.; Wasielewski, M. R. Phenothiazine radical cation excited states as super-oxidants for energy-demanding reactions. J. Am. Chem. Soc. 2018, 140 (15), 5290-5299.

(12) Fujitsuka, M.; Majima, T. Reaction dynamics of excited radical ions revealed by femtosecond laser flash photolysis. J. Photochem. Photobiol., C 2018, 35, 25-37.

(13) Bakulin, A. A.; Rao, A.; Pavelyev, V. G.; van Loosdrecht, P. H. M.; Pshenichnikov, M. S.; Niedzialek, D.; Cornil, J.; Beljonne, D.; Friend, R. H. The role of driving energy and delocalized states for charge separation in organic semiconductors. Science 2012, 335 (6074), 1340.

(14) Jakowetz, A. C.; Böhm, M. L.; Zhang, J.; Sadhanala, A.; Huettner, S.; Bakulin, A. A.; Rao, A.; Friend, R. H. What controls the rate of ultrafast charge transfer and charge separation efficiency in organic photovoltaic blends. J. Am. Chem. Soc. 2016, 138 (36), 11672-11679.

(15) Savoie, B. M.; Rao, A.; Bakulin, A. A.; Gelinas, S.; Movaghar, B.; Friend, R. H.; Marks, T. J.; Ratner, M. A. Unequal partnership: Asymmetric roles of polymeric donor and fullerene acceptor in generating free charge. J. Am. Chem. Soc. 2014, 136 (7), 2876-2884.

(16) Krainova, N.; Grede, A. J.; Tsokkou, D.; Banerji, N.; Giebink, N. C. Polaron photoconductivity in the weak and strong light-matter coupling regime. Phys. Rev. Lett. 2020, 124, 177401.

(17) Breslin, D. T.; Fox, M. A. Excited-state behavior of thermally stable radical ions. J. Phys. Chem. 1994, 98 (2), 408-411.

(18) Fujitsuka, M.; Kim, S. S.; Lu, C.; Tojo, S.; Majima, T. Intermolecular and intramolecular electron transfer processes from excited naphthalene diimide radical anions. J. Phys. Chem. B 2015, 119 (24), 7275-7282.

(19) Fujitsuka, M.; Ohsaka, T.; Majima, T. Dual electron transfer pathways from the excited $\mathrm{C}_{60}$ radical anion: enhanced reactivities due to the photoexcitation of reaction intermediates. Phys. Chem. Chem. Phys. 2015, 17 (46), 31030-31038.

(20) Voss, M. G.; Scholes, D. T.; Challa, J. R.; Schwartz, B. J. Ultrafast transient absorption spectroscopy of doped P3HT films: distinguishing free and trapped polarons. Faraday Discuss. 2019, 216 (0), 339-362.

(21) Becke, A. D. Density-functional thermochemistry. III. The role of exact exchange. J. Chem. Phys. 1993, 98 (7), 5648-5652.

(22) Lee, C.; Yang, W.; Parr, R. G. Development of the ColleSalvetti correlation-energy formula into a functional of the electron density. Phys. Rev. B: Condens. Matter Mater. Phys. 1988, 37 (2), 785789 .

(23) Miehlich, B.; Savin, A.; Stoll, H.; Preuss, H. Results obtained with the correlation energy density functionals of Becke and Lee, Yang and Parr. Chem. Phys. Lett. 1989, 157 (3), 200-206.

(24) Ditchfield, R.; Hehre, W. J.; Pople, J. A. Self-Consistent Molecular-Orbital Methods. IX. An extended gaussian-type basis for molecular-orbital studies of organic molecules. J. Chem. Phys. 1971, 54 (2), 724-728.

(25) Hehre, W. J.; Ditchfield, R.; Pople, J. A. Self-consistent molecular orbital methods. XII. Further extensions of gaussian-type basis sets for use in molecular orbital studies of organic molecules. $J$. Chem. Phys. 1972, 56 (5), 2257-2261.

(26) Gordon, M. S. The isomers of silacyclopropane. Chem. Phys. Lett. 1980, 76 (1), 163-168.

(27) Barone, V.; Cossi, M. Quantum calculation of molecular energies and energy gradients in solution by a conductor solvent model. J. Phys. Chem. A 1998, 102 (11), 1995-2001.
(28) Martin, R. L. Natural transition orbitals. J. Chem. Phys. 2003, 118 (11), 4775-4777.

(29) Frisch, M. J.; Trucks, G. W.; Schlegel, H. B.; Scuseria, G. E.; Robb, M. A.; Cheeseman, J. R.; Scalmani, G.; Barone, V.; Petersson, G. A.; Nakatsuji, H.; Li, X.; et al. Gaussian 16, Revision C.01; Gaussian, Inc.: Wallingford, CT, 2016.

(30) Tang, C. W.; VanSlyke, S. A. Organic electroluminescent diodes. Appl. Phys. Lett. 1987, 51 (12), 913-915.

(31) Tao, Y.; Yang, C.; Qin, J. Organic host materials for phosphorescent organic light-emitting diodes. Chem. Soc. Rev. 2011, 40 (5), 2943-2970.

(32) Borsenberger, P. M.; Bässler, H. Concerning the role of dipolar disorder on charge transport in molecularly doped polymers. J. Chem. Phys. 1991, 95 (7), 5327-5331.

(33) Kröger, M.; Hamwi, S.; Meyer, J.; Riedl, T.; Kowalsky, W.; $\mathrm{Kahn}, \mathrm{A}$. Role of the deep-lying electronic states of $\mathrm{MoO}_{3}$ in the enhancement of hole-injection in organic thin films. Appl. Phys. Lett. 2009, 95 (12), 123301.

(34) Greiner, M. T.; Helander, M. G.; Tang, W.-M.; Wang, Z.-B.; Qiu, J.; Lu, Z.-H. Universal energy-level alignment of molecules on metal oxides. Nat. Mater. 2012, 11 (1), 76-81.

(35) Meyer, J.; Hamwi, S.; Kröger, M.; Kowalsky, W.; Riedl, T.; Kahn, A. Transition metal oxides for organic electronics: Energetics, device physics and applications. Adv. Mater. 2012, 24 (40), 54085427.

(36) Tietze, M. L.; Benduhn, J.; Pahner, P.; Nell, B.; Schwarze, M.; Kleemann, H.; Krammer, M.; Zojer, K.; Vandewal, K.; Leo, K. Elementary steps in electrical doping of organic semiconductors. Nat. Commun. 2018, 9 (1), 1182.

(37) Kalinowski, J.; Giro, G.; Cocchi, M.; Fattori, V.; Di Marco, P. Unusual disparity in electroluminescence and photoluminescence spectra of vacuum-evaporated films of 1,1-bis ((di-4-tolylamino) phenyl) cyclohexane. Appl. Phys. Lett. 2000, 76 (17), 2352-2354.

(38) Sasaki, Y.; Araki, Y.; Fujitsuka, M.; Ito, O.; Hirao, A.; Nishizawa, $\mathrm{H}$. Photoinduced electron transfer and electron-mediating systems from aromatic amines to triplet states of $\mathrm{C}_{60}$ and $\mathrm{C}_{70}$ in the presence of a viologen dication. Photochem. Photobiol. Sci. 2003, 2 (2), 136-141.

(39) Causa', M.; Ramirez, I.; Martinez Hardigree, J. F.; Riede, M.; Banerji, N. Femtosecond dynamics of photoexcited $\mathrm{C}_{60}$ films. J. Phys. Chem. Lett. 2018, 9 (8), 1885-1892.

(40) Moore, G. J.; Causa', M.; Martinez Hardigree, J. F.; Karuthedath, S.; Ramirez, I.; Jungbluth, A.; Laquai, F.; Riede, M.; Banerji, N. Ultrafast charge dynamics in dilute-donor versus highly intermixed TAPC: $\mathrm{C}_{60}$ organic solar cell blends. J. Phys. Chem. Lett. 2020, 11 (14), 5610-5617.

(41) Cheng, C.-Y.; Dhanker, R.; Gray, C. L.; Mukhopadhyay, S.; Kennehan, E. R.; Asbury, J. B.; Sokolov, A.; Giebink, N. C. Charged polaron polaritons in an organic semiconductor microcavity. Phys. Rev. Lett. 2018, 120 (1), 017402.

(42) Cheng, C.-Y.; Kim, H.; Giebink, N. C. Charged polariton luminescence from an organic semiconductor microcavity. ACS Photonics 2019, 6 (2), 308-313.

(43) Lee, J.-H.; Kim, H.-M.; Kim, K.-B.; Kabe, R.; Anzenbacher, P.; Kim, J.-J. Homogeneous dispersion of organic p-dopants in an organic semiconductor as an origin of high charge generation efficiency. Appl. Phys. Lett. 2011, 98 (17), 173303.

(44) Glaser, T.; Beck, S.; Lunkenheimer, B.; Donhauser, D.; Köhn, A.; Kröger, M.; Pucci, A. Infrared study of the $\mathrm{MoO}_{3}$ doping efficiency in 4,4'-bis(N-carbazolyl)-1,1'-biphenyl (CBP). Org. Electron. 2013, 14 (2), 575-583.

(45) Tauler, R. Multivariate curve resolution applied to second order data. Chemom. Intell. Lab. Syst. 1995, 30 (1), 133-146.

(46) Jaumot, J.; Gargallo, R.; de Juan, A.; Tauler, R. A graphical userfriendly interface for MCR-ALS: a new tool for multivariate curve resolution in MATLAB. Chemom. Intell. Lab. Syst. 2005, 76 (1), 101110.

(47) Awartani, O. M.; Gautam, B.; Zhao, W.; Younts, R.; Hou, J.; Gundogdu, K.; Ade, H. Polymer non-fullerene solar cells of vastly 
different efficiencies for minor side-chain modification: impact of charge transfer, carrier lifetime, morphology and mobility. J. Mater. Chem. A 2018, 6 (26), 12484-12492.

(48) Etzold, F.; Howard, I. A.; Forler, N.; Melnyk, A.; Andrienko, D.; Hansen, M. R.; Laquai, F. Sub-ns triplet state formation by nongeminate recombination in PSBTBT:PC ${ }_{70} B M$ and PCPDTBT:PC ${ }_{60} \mathrm{BM}$ organic solar cells. Energy Environ. Sci. 2015, 8 (5), 1511-1522.

(49) Grilj, J.; Buchgraber, P.; Vauthey, E. Excited-state dynamics of Wurster's salts. J. Phys. Chem. A 2012, 116 (28), 7516-7522.

(50) Brodard, P.; Sarbach, A.; Gumy, J.-C.; Bally, T.; Vauthey, E. Excited-state dynamics of organic radical Ions in liquids and in lowtemperature matrices. J. Phys. Chem. A 2001, 105 (27), 6594-6601.

(51) Grilj, J.; Laricheva, E. N.; Olivucci, M.; Vauthey, E. Fluorescence of radical ions in liquid solution: Wurster's Blue as a case study. Angew. Chem., Int. Ed. 2011, 50 (19), 4496-4498.

(52) Grilj, J.; Zonca, C.; Daku, L. M. L.; Vauthey, E. Photophysics of the galvinoxyl free radical revisited. Phys. Chem. Chem. Phys. 2012, 14 (18), 6352-6358.

(53) Englman, R.; Jortner, J. The energy gap law for radiationless transitions in large molecules. Mol. Phys. 1970, 18 (2), 145-164.

(54) Tokmachev, A. M.; Boggio-Pasqua, M.; Bearpark, M. J.; Robb, M. A. Photostability via sloped conical intersections: A computational study of the pyrene radical cation. J. Phys. Chem. A 2008, 112 (43), 10881-10886.

(55) Chekalin, S. V.; Yartsev, A. P.; Sundström, V. The primary stages of the charge carrier photogeneration in $\mathrm{C}_{60}$ films studied by the 100-fs laser pulse pump-probe method. J. Exp. Theor. Phys. 2001, 93 (4), 706-716.

(56) Causa', M.; De Jonghe-Risse, J.; Scarongella, M.; Brauer, J. C.; Buchaca-Domingo, E.; Moser, J.-E.; Stingelin, N.; Banerji, N. The fate of electron-hole pairs in polymer:fullerene blends for organic photovoltaics. Nat. Commun. 2016, 7 (1), 12556.

(57) Gélinas, S.; Rao, A.; Kumar, A.; Smith, S. L.; Chin, A. W.; Clark, J.; van der Poll, T. S.; Bazan, G. C.; Friend, R. H. Ultrafast longrange charge separation in organic semiconductor photovoltaic diodes. Science 2014, 343 (6170), 512.

(58) Boxer, S. G. Stark realities. J. Phys. Chem. B 2009, 113 (10), $2972-2983$.

(59) Bublitz, G. U.; Boxer, S. G. Stark spectroscopy: Applications in chemistry, biology, and materials science. Annu. Rev. Phys. Chem. 1997, 48 (1), 213-242.

(60) Lu, C.; Fujitsuka, M.; Sugimoto, A.; Majima, T. Unprecedented intramolecular electron transfer from excited perylenediimide radical anion. J. Phys. Chem. C 2016, 120 (23), 12734-12741.

(61) Marcus, R. A.; Sutin, N. Electron transfers in chemistry and biology. Biochim. Biophys. Acta, Rev. Bioenerg. 1985, 811 (3), 265322.

(62) Marcus, R. A. Electron Transfer Reactions in Chemistry: Theory and Experiment (Nobel Lecture). Angew. Chem., Int. Ed. Engl. 1993, 32 (8), 1111-1121. 